\title{
A Game theoretic approach for competition over visibility in social networks
}

\author{
Khadija Touya, Mohamed Baslam, Rachid El Ayachi, Mostafa Jourhmane \\ TIAD Laboratory, Sultan Moulay Slimane University, Morocco
}

\begin{tabular}{l}
\hline \hline Article Info \\
\hline Article history: \\
Received Sep 14, 2017 \\
Revised Jan 17, 2018 \\
Accepted Jan 7, 2019 \\
\hline
\end{tabular}

\section{Keywords:}

Best response

Concave game

Game theory

Nash equilibrium

Price of anarchy

Social networks

\begin{abstract}
Social Networks have known an important evolution in the last few years. These structures, made up of individuals who are tied by one or more specific types of interdependency, constitute the window for members to express their opinions and thoughts by sending posts to their own walls or others' timelines. Actually, when a content arrives, it's located on the top of the timeline pushing away older messages. This situation causes a permanent competition over visibility among subscribers who jump on opponents to promote conflict. Our study presents this competition as a non-cooperative game; each source has to choose frequencies which assure its visibility. We model it, exploring the theory of concave games, to reach a situation of equilibrium; a situation where no player has the ultimate ability to deviate from its current strategy. We formulate the named game, then we analyze it and prove that there is exactly one Nash equilibrium which is the convergence of all players' best responses. We finally provide some numerical results, taking into consideration a system of two sources with a specific frequency space, and analyze the effect of different parameters on sources' visibility on the walls of social networks.
\end{abstract}

Copyright ( 12019 Institute of Advanced Engineering and Science. All rights reserved.

\section{Corresponding Author:}

Khadija Touya,

TIAD Laboratory,

Sultan Moulay Slimane University, Morocco.

Email: k.touya@usms.ma

\section{INTRODUCTION}

The rise of the internet came up with a digital technique permitting to construct social and active environments of interactions and relationships (Facebook, Twitter, Linkedin...). Those environments connect social actors called nodes, with each other by certain types of interdependency such as: friendship, common interest, financial exchange, dislikes, relationships of beliefs and knowledge.

Moreover, these structures, which are considered as sets of participants (individuals, organizations...), are changing the way of interacting via Internet [1]. To be one of the users and interact with others, a source has to share some personal information but also to post messages on timelines. Therefore, there are many factors impacting the visibility of all the arrival posts, such as: timelines' limited capacity, visibility limitation, the past behavior of a user on a social network and number of likes. In fact, each one of the cited factors influences the visibility in many ways. To overcome these limitations, selfish sources have to post more contents in order to keep themselves visible on a specific wall. Henceforth, this selfishness leads to a competition over visibility.

To analyze this kind of competition and model it, a variety of techniques took place, including those from network science and game theory. However, a game theoretic approach can offer new views beyond what classical methods could do. This mathematical discipline, interested in strategic decision-making, modeling and analyzing interactions among individuals is launched on 1944 by John Von Neumann 
(mathematician) and Morgenstern (economist) who justified the idea to maximize the expected payoff in their work in [2] and followed by contributions of Nash (1950) [3].

This theory is a basic tool, which is used in many disciplines [4] among whom we can name: economy [2], Biology [5, 6], Planning of Generation and Transmission [7], Ad-hoc networks [8], cognitive radio networks [9] and road traffic [10], It is also applied in telecommunication networks [11, 12] to analyze situations and find a point of equilibrium (Price and Quality of Service) among SPs.

Hence, the purpose of the named theory is to balance the interactions between players, to predict and define the possible outcomes of such interactions, and to develop strategies of players which lead to an optimal outcome of the game. One of the principle keys of the game theory is the dependence of the players' actions on the choices of others, more than their own choices.

The main topic of this work is to perform behavior modeling in social networks using the theory of concave games which is introduced by J. Rosen in [13] assuring an interesting analysis for the existence and the uniqueness of the equilibrium point of a game. Following [13], we aim to develop an efficient approach which gives a situation of equilibrium solving the competition over visibility on social networks' timelines.

In this context, (A. Reiffers-Masson et al 2014) established a previous paper [14]. This work was about presenting an approach to model the competition over visibility in social networks employing the famous game theory. In order to accomplish this study, authors aimed to model the problem of selecting the content creation's rates as a noon cooperative game. They analyzed and reduced it to the well-known generalized Kelly mechanism allowing defining and extracting the equilibrium between various sources taking into account several groups and the possibility for a message to be relayed from one group to another, but also, authors proved the existence of this equilibrium.

Furthermore, many researches have been conducted in applying the game theory in social networks, these works were established to study and model the behavior of sources to reach a particular objective [15-17].

To reach the objective of our theoretic game approach, we organized the rest of this paper as follows: in section 2, we propose the study and analysis of the non-cooperative game between selfish and competitive information providers, this analysis consists of employing algorithmic game theory especially the algorithm of Best Response converging to the Nash equilibrium. In section 3, we present obtained results illustrating and assisting our study. Finally, we sum up with a general conclusion opening the window for future works.

\section{COMPETITION MODELING}

This section is reserved to model the interaction among information providers (IP), sharing some common destination to which they send contents or post messages, as a non-cooperative game. In other words, sending new posts makes the older ones desperate from the timeline and pushes sources who are assumed to be rational to resend their posts and share more messages. Further, this behavior is producing a competition concerning the visibility on the walls of social networks.

This problematic leads us to think about the theory of concave games, which seems to be the efficient tool for analyzing this kind of competition situation. In order to attend this objective, we have to specify the number of players (sources), the possible actions for each player, constraints which may be imposed on them and the objective function of each player. In fact, each source chooses the appropriate strategy (depending on other sources' strategies) to guarantee its visibility on a feed news. It has also a cost to send messages; this cost depends on its own frequency.

In this context, we consider a system with $\mathrm{K}$ information providers. Let $\lambda \mathrm{j}$ and $\lambda$-j be, respectively, the publishing frequencies of a player $\mathrm{j}$ and other players, and $\gamma \mathrm{j}$ the cost a player $\mathrm{j}$ has for sending posts on a wall of a social network.

Let $\mathrm{G}=[\mathrm{K},\{\lambda \mathrm{j}\},\{\mathrm{Uj}()\}$.$] denotes the non-cooperative frequency game, where \mathrm{K}$ identifies a number of Information Providers,$\lambda j$ is the frequency strategy set of IPj, and $\mathrm{Uj}_{\mathrm{j}}$.) is the utility function the IPj has to maximize by choosing a convent frequency $\lambda \mathrm{j}$. Formally, the source's $\mathrm{j}$ objective function is defined as follows:

$$
U_{j}\left(\lambda_{j}, \lambda_{-j}\right)=K \frac{\lambda_{j}}{\sum_{i} \lambda_{i}}-\lambda_{j} \gamma_{j}
$$

Where $\lambda-\mathrm{j}=(\lambda 1, \ldots ., \lambda \mathrm{j}-1, \lambda \mathrm{j}+1, \ldots ., \lambda \mathrm{K})$ is the strategy vector of the other IPs.

\section{Assumption 1}

To achieve the analysis, we adopt the following assumption: 
$\lambda_{j} \geq 1, \forall j \in K$

This hypothesis means that each source publishes at least once, which is realistic. Therefore, sources that do not publish won't be taken into account.

\subsection{The Nash equilibrium}

Considering the competition among selfish IPs, the natural solution of this non-cooperative game is gave by the Nash equilibrium that Nash produced in [3]. This equilibrium point is defined as a strategy profile such that no provider can unilaterally increase his revenue [18]. To demonstrate the existence and the uniqueness of the Nash equilibrium, we explore the concave games' theory [13]. We recall that a non-cooperative game $\mathrm{G}$ is called concave if all players' utility functions are strictly concave with respect to their corresponding strategies [13].

According to [13], a Nash equilibrium exists in a concave game if the joint strategy space is compact and convex, and the utility function that any given player seeks to maximize is concave in its own strategy and continuous at every point in the product strategy space.

\section{Definition 1}

A frequency vector $\lambda^{*}=\left(\lambda^{*}{ }_{1}, \ldots, \lambda^{*}\right)$ is a Nash equilibrium if for every $\mathrm{j} \in\{1, \ldots, \mathrm{K}\}$,

$$
U_{j}\left(\lambda_{j}^{*}, \lambda_{-j}^{*}\right)=\max _{\lambda_{j} \in\left[1, \lambda_{j}\right]} U_{j}\left(\lambda_{j}, \lambda_{j}^{*}\right)
$$

Where $\lambda_{\max }$ is the maximum frequency.

In other way, reaching the equilibrium point, no source could get a profit by changing its strategy in an unilateral manner.

\section{Theorem 1}

For the game $\mathrm{G}$ which is concave the Nash equilibrium exists and it's unique.

\section{Proof 1}

To prove the existence of the Nash equilibrium, it is sufficient to show the negativity of the second derivative of $U_{j}(\forall j)$.

$$
\frac{\partial U_{j}\left(\lambda_{j}, \lambda_{-j}\right)}{\partial \lambda_{j}}=K \frac{\sum_{i} \lambda_{i}-\lambda_{j}}{\left(\sum_{i} \lambda_{i}\right)^{2}}-\gamma_{j}
$$

This produces the second derivative, given as follows:

$$
\frac{\partial^{2} U_{j}\left(\lambda_{j}, \lambda_{-j}\right)}{\partial \lambda_{j}^{2}}=-2 \frac{K}{\left(\sum_{i} \lambda_{i}\right)^{2}}\left(1-\frac{\lambda_{j}}{\sum_{i} \lambda_{i}}\right)
$$

The utility function is concave if the result obtained in (2) is strictly negative. To ensure this concavity, we have to verify the following condition:

$$
1-\frac{\lambda_{j}}{\sum_{i} \lambda_{i}}>0
$$

According to the assumption $1, \lambda_{j} \geq 1$. So the last condition is verified and we obtain the following result:

$$
\frac{\partial^{2} U_{j}\left(\lambda_{j}, \lambda_{-j}\right)}{\partial \lambda_{j}^{2}}<0
$$

We finally conclude that the game we formulated is concave and this concavity ensures the existence of the Nash Equilibrium.

Now we turn to discuss the uniqueness of the existent Nash Equilibrium. We must calculate the second derivative of $U_{j}$ with respect to $\lambda_{j}$ and $\lambda_{-j}$ and verify its test as follows: 
$\frac{\partial^{2} U_{j}\left(\lambda_{j}, \lambda_{-j}\right)}{\partial \lambda_{j}^{2}}<\sum_{l \neq j} \frac{\partial^{2} U_{j}\left(\lambda_{j}, \lambda_{-j}\right)}{\partial \lambda_{j} \partial \lambda_{l}}$

Taking into consideration the $\mathrm{U}_{\mathrm{j}}$ defined in (1), we compute its first derivative as follows:

$$
\frac{\partial U_{j}\left(\lambda_{j}, \lambda_{-j}\right)}{\partial \lambda_{l}}=-K \frac{\lambda_{j}}{\left(\Sigma_{i} \lambda_{i}\right)^{2}}
$$

So, the second derivative is determined to be:

$$
\begin{aligned}
\frac{\partial^{2} U_{j}\left(\lambda_{j}, \lambda_{-j}\right)}{\partial \lambda_{j} \partial \lambda_{l}} & =-\frac{K}{\left(\sum_{i} \lambda_{i}\right)^{2}}\left(1-2 \frac{\lambda_{j}}{\sum_{i} \lambda_{i}}\right) \\
& =-\frac{2 K}{\left(\sum_{i} \lambda_{i}\right)^{2}}\left(\frac{1}{2}-\frac{\lambda_{j}}{\sum_{i} \lambda_{i}}\right)
\end{aligned}
$$

Or

$$
-\frac{2 K}{\left(\sum_{i} \lambda_{i}\right)^{2}}\left(\frac{1}{2}-\frac{\lambda_{j}}{\sum_{i} \lambda_{i}}\right)=-2 \frac{K}{\left(\sum_{i} \lambda_{i}\right)^{2}}\left(1-\frac{\lambda_{j}}{\sum_{i} \lambda_{i}}\right)+\frac{K}{\left(\Sigma_{i} \lambda_{i}\right)^{2}}
$$

That produces

$$
\frac{\partial^{2} U_{j}\left(\lambda_{j}, \lambda_{-j}\right)}{\partial \lambda_{j} \partial \lambda_{l}}=\frac{\partial^{2} U_{j}\left(\lambda_{j}, \lambda_{-j}\right)}{\partial \lambda_{j}^{2}}+\frac{K}{\left(\Sigma_{i} \lambda_{i}\right)^{2}}
$$

Therefore, the sum of these derivatives is obtained as:

$$
\sum_{l \neq j} \frac{\partial^{2} U_{j}\left(\lambda_{j}, \lambda_{-j}\right)}{\partial \lambda_{j} \partial \lambda_{l}}=\sum_{l \neq j}\left(\frac{\partial^{2} U_{j}\left(\lambda_{j}, \lambda_{-j}\right)}{\partial \lambda_{j}^{2}}+\frac{K}{\left(\sum_{i} \lambda_{i}\right)^{2}}\right)
$$

Or (3) shows that the second derivative of the utility function is strictly negative, $\forall \lambda_{j} \geq 1$, and $\frac{K}{\left(\Sigma_{i} \lambda_{i}\right)^{2}}>0$

Using the result reached in (5), we prove the test presented in (4). Hence, the uniqueness of the Nash equilibrium is guaranteed.

\subsection{Convergence to the Nash equilibrium: algorithm of the best response}

The demonstration of the existence of a unique Nash equilibrium leads to the convergence study using the algorithmic game theory, a fascinating fusions of ideas of both fields-game theory and algorithms-, [16], as algorithms became the natural environment and default platform of strategic decision making. The particular task of this work is to employ one of the most known algorithms in game theory; the Best Response Algorithm in order to learn the equilibrium's parameter. This Algorithm defines a best response sequence as a couple $(t(n), j(n))$, where $t(n)$ is a strictly increasing sequence of times such that at time $t(n)$ player $\mathrm{j}(\mathrm{n})$ updates its action using a best response to the current actions of the other players. Therefore, there is a unique equilibrium point and any sequence of best responses of the players converges to it in a finite time.

As shown in (3), the utility functions $U_{j}\left(\lambda_{j}, \lambda_{-j}\right)$ are concave. Therefore, for each user $j$, the the frequency maximizing its utility is the solution of the following equation:

$$
\frac{\partial U_{j}\left(\lambda_{j}, \lambda_{-j}\right)}{\partial \lambda_{j}}=0
$$

The resolution of the last equation introduced the best response in term of frequency $\mathrm{BR}_{\lambda(\text { (.) }}^{\mathrm{j}}$ of each source $\mathrm{j}$ as a function of its opponents' strategies. We define this notion as following:

$$
B R=\operatorname{argmax} U_{j}\left(\lambda_{j}, \lambda_{-j}\right)
$$

As already seen, a sequence of best responses converges to the Nash equilibrium since this one is unique. Algorithm 1 details the work done using the BR [11].

Algorithm 1: Best Response 
Initialization of the frequency vector $\lambda$;

For each source $j \in K$ at iteration $t$ :

$$
\lambda_{j}^{t+1}=B R_{\lambda}^{j}\left(\lambda^{t}\right)
$$

This algorithm aims to compute the best response that maximizes the utility function. When the $\operatorname{BR}_{\lambda}{ }^{j}\left(\lambda^{t-1}\right)=\operatorname{BR}_{\lambda}{ }^{j}\left(\lambda^{t}\right)=B{ }^{j}{ }^{j}\left(\lambda^{t+1}\right)$, we conclude that the algorithm has converge to the equilibrium point.

\subsection{Price of anarchy}

Papadimitriou, in [19], introduced the term of "the price of anarchy" and then multiple kinds of works have been produced around this concept, especially, the book [20] developing central ideas of it. Furthermore, this important measure of loss of equilibria's efficiency has come more popular due to the work of Roughgarden and Tardos [21] where they opened the window for studying the price of anarchy in atomic and nonatomic congestion games. It has been also studied in [22] for network creation and in [23] for facility location.

Seeming to be the most popular measure of the inefficiency of the equilibrium, the price of anarchy resolves the issue of multiple equilibrium adopting a worst-case approach; an approach defining that loss as the worst-case ratio comparing the global efficiency measure at an outcome, to the optimal value of that efficiency measure. In other words, in a noncooperative game, the price of anarchy is considered as the ratio between the worst utility function value of an equilibrium and the one of an optimal outcome.

According to [20], the inefficiency caused by the player's selfishness is measured as the quotient between the social welfare, that Maille and Tuffin presented as the sum of the utilities of all providers in the system) in [24], obtained at the Nash equilibrium and the maximum value of the social welfare, as shown in (6)

$$
P O A=\frac{\min W_{N E}(\lambda)}{\max W(\lambda)}
$$

Where, $\max W(\lambda)=\max \sum_{j=1}^{K} U_{j}(\lambda)$ is a welfare function, and $W_{N E}(\lambda)=\sum_{j=1}^{K} U_{j}\left(\lambda^{*}\right)$ is a sum of utilities of all agents at a Nash equilibrium.

If there are many equilibrium points, we turn to adopt the worst case of the equilibrium, so we use $\min W_{N E}(\lambda)=\min \sum_{j=1}^{K} U_{j}\left(\lambda^{*}\right)$.

\section{RESULTS AND ANALYSIS}

In this paper, we report on what we consider a noticeable application of game theory to model competition over visibility in social networks. To clarify our analysis, we suggest to study numerically the equilibrium frequency $\lambda^{*}$ by considering the utility functions of sources as well as the best response of each one. Hence, we consider a social network with two sources $K=2$ trying to maximize their respective revenues in term of visibility in this system. The frequency strategies' set is assumed to be $\lambda_{\mathrm{j}}=[1,80]$ and the parameter $\gamma_{j}$, which is the indicative of the cost paid by the competitors to operate at higher frequencies, is the same for both sources. The specification of the cost's value must take into consideration that the utility function has to be positive for each set of frequencies.

$$
U_{j}\left(\lambda_{j}, \lambda_{-j}\right)>0, \forall \lambda_{j} \in[1,80]
$$

Given that $\lambda=\left(\lambda_{\mathrm{j}}, \lambda_{-\mathrm{j}}\right)=(15,1)$ maximizes the $\mathrm{U}_{\mathrm{j}}$, the cost we are searching for is the solution of the following equation:

$$
\frac{\partial U_{j}\left(\lambda_{j}, \lambda_{-j}\right)}{\partial \lambda_{j}}=0
$$

It gives that:

$K \frac{\sum_{i} \lambda_{i}-\lambda_{j}}{\left(\sum_{i} \lambda_{i}\right)^{2}}-\gamma_{j}=0$

Replacing the frequencies with their values, we obtain the cost's value, which is: 
$\gamma_{j}=2 *\left(\frac{1}{(16)^{2}}\right)=\frac{2}{256}$

Taking into account that the named players have a symmetric behavior, in the rest of this section, we will study their visibility with respect to the different parameters of the considered system and present the obtained results only for one source hence it is the same for the other player.

* Fixed cost $\gamma_{j}=2 / 256$

Assuming that sending messages on a wall comes with a fixed cost, we firstly plot, in Figure 1, the utility function of the player $\mathrm{j}$ considering that $\lambda_{1}$; the other player's frequency; is equal to $1\left(\lambda_{1}=1\right)$.

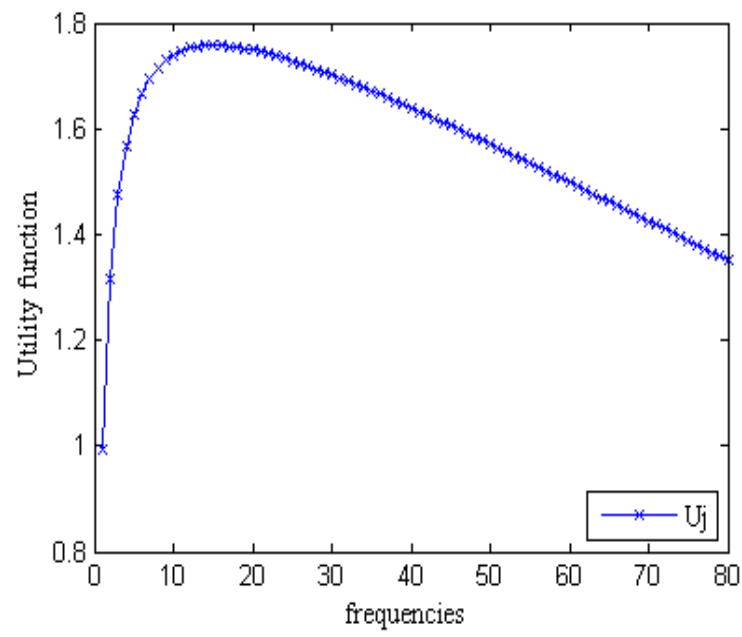

Figure 1. Function utility of player $\mathrm{j}$ when $\lambda \mathrm{l}=1$

According to the graph, we remark that $\mathrm{U}_{\mathrm{j}}$ is a concave function. Henceforth, the existence and uniqueness of the equilibrium situation are guaranteed. We will thus present the best responses for both sources in Figure 2.

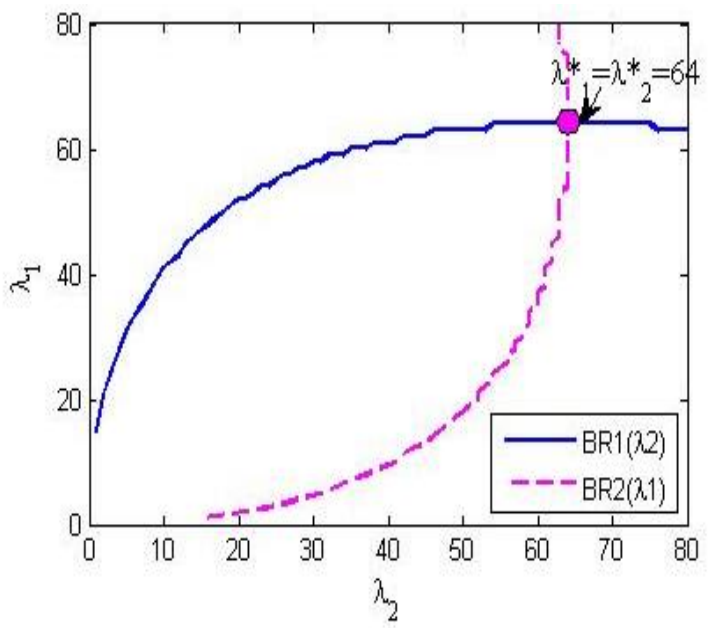

Figure 2. Best responses for both sources

The presentation of the BRs revels a point of intersection; this point constitutes a point of equilibrium among the considered sources. It is numerically showed to be $\lambda *_{1}=\lambda *_{2}=64$. Figure 3 represents the curve of the convergence to the Frequency Nash Equilibrium; this convergence is assured using the Best Response algorithm (algorithm 1). 


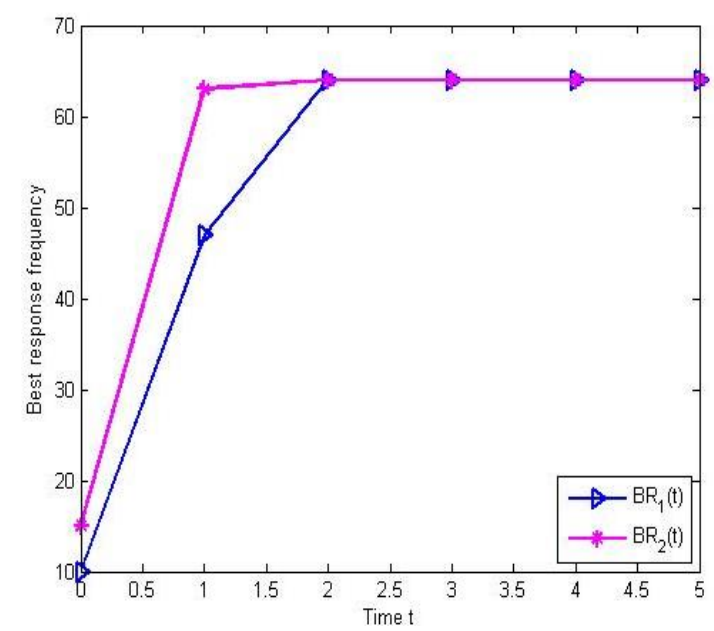

Figure 3. Convergence to the frequency Nash equilibrium

We remark that the sequence of best responses converges to the unique Frequency Nash Equilibrium (it seems to be the same value found on Figure 2), but also this convergence is highly speed (around four rounds are enough to attend the equilibrium point).

\section{* Cost $\gamma_{\mathrm{j}=}[2 / 256,50 / 256]$}

In this part, we'll compute the frequency's sets at the equilibrium and present the utility functions of each source $\mathrm{j}$ with respect to several values of posting messages' cost.

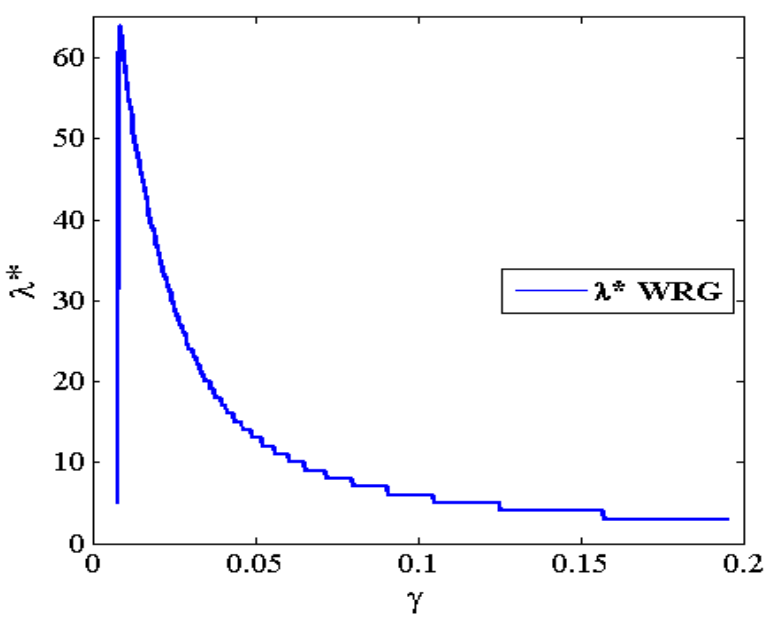

Figure 4. Evolution of $\lambda$ at the equilibrium WR to $\gamma_{j}$

According to the graph presented in Figure 4, we conclude that the algorithm employed to accomplish our work has converged to the equilibrium for each value of the cost $\gamma_{j}$. We remark also that when the cost increases the set of frequencies assuring the equilibria knows a decreasing evolution. The Figure 5 revels the values of utility functions for each source, these values has an evolution in a decreasing manner with respect to the cost. The evolution of $U_{j}$ follows the decreasing values of the frequencies' set obtained at the equilibrium. To measure the inefficiency of the equilibrium, we run to study the variation of the price of anarchy as a function of the cost $\gamma$ in Figure 6. 


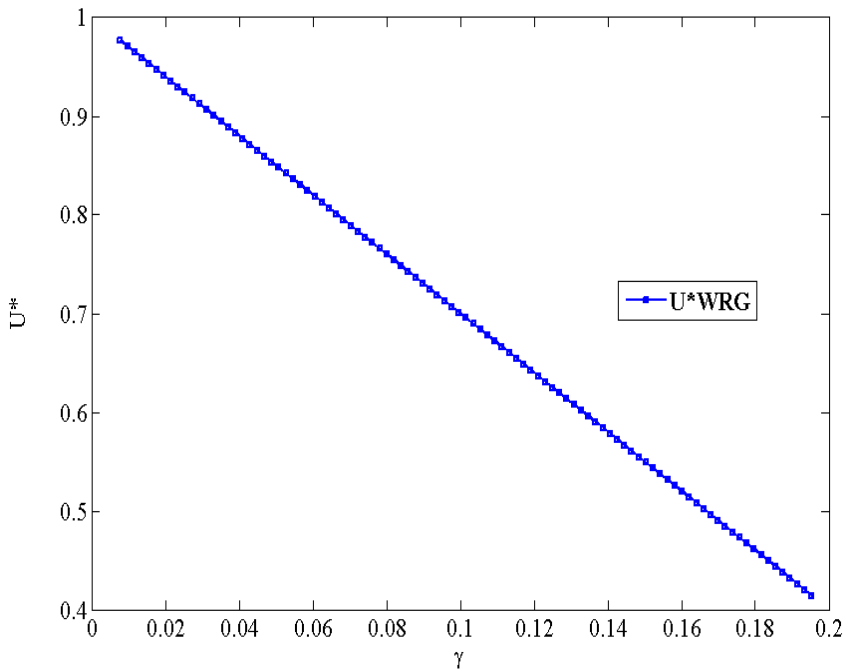

Figure 5. Utility functions at the equilibrium WR to $\gamma$

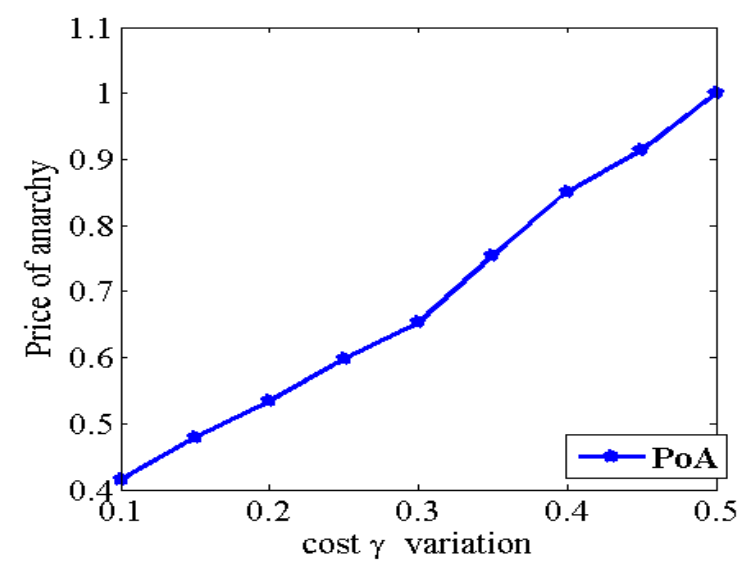

Figure 6. Price of anarchy as a function of the cost $\gamma$

The curve reveals that the Nash equilibrium of this game is fair and socially efficient since the PoA is close to 1.Therefore, these results reveal that the proposed model has reached the aim we specified in the beginning of this work. To generalize the reached results, we take into consideration that inside a system constituted by $\mathrm{K}$ sources, the interaction is the same between each couple of sources. Henceforth, the equilibrium exists, it is unique and socially efficient in a game with $\mathrm{K}$ sources and the obtained results assure the aim of our theoretical approach.

\section{CONCLUSION}

In this paper, we were concerned with a situation where many participants interact; a system where sources try their best and compete to keep their visibility on timelines. Giving that such a situation is naturally modeled by games that involve many players, we aimed to analyze this sort of competitions as a non-cooperative game. We took into consideration that the utility function of a user depends on his frequency of sending posts on the wall of a social network but also on other users' frequencies, and posting messages comes with a cost, which is proportional for its frequency. We formulated the named game using the theory of concave games to which the Nash equilibrium seemed to be the efficient solution and studied this efficiency employing the price of anarchy. Concerning the numerical investigations, we considered a social network containing 2 sources competing over visibility, we explored the best response algorithm to compute the equilibrium which is found in a finite number of iterations and present the results obtained concerning the 
frequencies and the utility functions at the equilibrium for each source of information. We finally presented the variation of the PoA, which assured the efficiency of the found equilibria.

\section{REFERENCES}

[1] K. Curran, S. Morrison, S. Mc Cauley, "Google+ vs Facebook: The Comparison", TELKOMNIKA Telecommunication, Computing, Electronics and Control, 2012; 10(2): 379-388.

[2] V. Neumann and J. Morgenstern, "Theory of games and economic behavior", Princeton University Press, 1944.

[3] N. Nisan, "Algorithmic game theory", Cambridge University Press, 2007.

[4] E. Altman and R. El Azouzi, "Theory of non-cooperative games applied in telecommunication networks", 2007.

[5] M.Smith, "Game theory and the evolution of fighting", In John Maynard Smith, editor, On Evolution (Edinburgh: Edinburgh University Press), 1972.

[6] M. Smith, "Evolution and the Theory of Games", Cambridge University Press, Cambridge, UK, 1982.

[7] M. Zareian Jahromi, et al, "A Secure-Coordinated Expansion Planning of Generation and Transmission Using Game Theory and Minimum Singular Value", International Journal of Electrical and Computer Engineering (IJECE), 2014: 4(6); 868-881.

[8] A. Sahnoun, et al, "A Coalition Formation Game Model for Energy Efficient Routing in Mobile Ad-hoc Network", International Journal of Electrical and Computer Engineering (IJECE), 2018; 8(1): 26-33.

[9] S. Lim, "Game Theoretic Channel Allocation in Cognitive Radio Networks", International Journal of Electrical and Computer Engineering (IJECE), 2017; 7(2): 986-991.

[10] J. G.Wardrop, "Some theoretical aspects of road traffic research communication networks", Proc. Inst. Civ. Eng., 1952.

[11] M. Baslam, et al, "Joint Price and QoS Market Share Game with Adversarial Service Providers and Migrating Customers", Proc. of Game Nets, Shanghai, China, April 2011.

[12] R. El Azouzi, et al, "Telecommunications Network Equilibrium with Price and Quality-of-Service Characteristics", Proc. of ITC, Berlin, Sept 2003.

[13] J. Rosen, "Existence and uniqueness of equilibrium points for concave n-person games", Econometrica, 1965.

[14] A. Reiffers-Masson, et al, "Game theory approach for modeling competition over visibility on social networks", Social Networking Workshop, India, 2014.

[15] E. Altman, "A stochastic game approach for competition over popularity in social networks", HAL INRIA, 2012

[16] E. Altman, "Game theoretic approaches for studying competition over popularity and over advertisement space in social networks", Value tools, 2012.

[17] E. Altman., et al, "Competition Over Timeline in Social Networks". Proceedings of the 2013 IEEE/ACM International Conference on Advances in Social Networks Analysis and Mining, 2013: 1352-1357.

[18] L. Hui, A Novel QoS Routing Algorithm in Wireless Mesh Networks, Indonesian Journal of Electrical Engineering and Computer Science (IJEECS), 2013: 11(3): 1652-1664.

[19] K. Koutsoupias and C. H. Papadimitriou, "Worst-case equilibria", In Proceedings of the 16th Annual Symposium on Theoretical Aspects of Computer Science, pages 404-413, 1999.

[20] L. Guijarro, V. Pla, J. R. Vidal and J. Martinez-Bauset, "Analysis of price competition under peering and transit agreements in Internet Service provision to peer-to-peer users," 2011 IEEE Consumer Communications and Networking Conference (CCNC), Las Vegas, NV, 2011, pp. 1145-114

[21] T. Roughgarden andE. Tardos, "How bad is selfish routing?", In Proceedings of the 41st Annual Symposium on Foundations of Computer Science, pages 93-102, Redondo Beach, CA, November 12-14, 2000.

[22] E. Anshelevich, et al, "Near-optimal network design with selfish agents", Theory of Computing, p: 77-109, 2008.

[23] A. Vetta, "Nash equilibria in competitive societies with application to facility location", In Proc. 43th Symp. Foundations of Computer Science (FOCS) p. 416, 2002.

[24] P. Maille and B. Tuffin, "Analysis of Price Competition in a Slotted Resource Allocation Game," IEEE INFOCOM 2008-The 27th Conference on Computer Communications, Phoenix, AZ, 2008, pp. 888-896. 